UDC $517.9+531.19$

A. V. Sinitsyn (Univ. Nac. de Colombia, Bogotá, Colombia;

Inst. System Dynamics and Control Theory SB RAS, Irkutsk, Russia),

E. V. Dulov (Univ. Nac. de Colombia, Bogotá, Colombia)

\title{
ON A THEORETICAL STUDY
}

FOR THE SOLUTION PROPERTIES OF THE LIMIT PROBLEM

FOR THE MAGNETICALLY NONINSULATED DIODE*

\section{ПРО ТЕОРЕТИЧНЕ ДОСЛІДЖЕННЯ \\ ВЛАСТИВОСТЕЙ РОЗВ'ЯЗКІВ ГРАНИЧНОЇ ЗАДАЧІ ДЛЯ МАГНІТНО НЕІЗОЛЬОВАНОГО ДІОДА}

In this paper we study the lower and upper bounds for the solutions of the limit problem of the plane vacuum diod in the magnetic field in the statement by N. Ben Abdallah, P. Degond and F. M'ehats. This problem was finally set by a physists in late 1980-s and was attentively studied by a number of the mathematitians in 1990-s.

Досліджено нижні і верхні межі для розв'язків граничної задачі плоского вакуумного діода у магнітному полі у постановці Н. Бен Абдалла, П. Дегонда та Р. Мехаца. Ця задача була остаточно поставлена фізиками наприкінці 1980-х років і уважно досліджена багатьма математиками у 1990-х роках.

1. Introduction. This paper is aimed at studying the stationary self-consistent problem of magnetic insulation under space-charge limitation via the asymptotics of the VlasovMaxwell system. This approach has been introduced by Langmuir and Compton [1] and recently developed by Degond and Raviart [2], N. Ben Abdallah, P. Degond and F. M'ehats [3] to analyze the space charge limited operation of a vacuum diode. In a dimensionless form of the Vlasov-Poisson system, the ratio of the typical particle velocity at the cathode to that reached at the anode appears as a small parameter [2]. The associated perturbation analysis provides a mathematical framework to the results of Langmuir and Compton [1], stating that the current flowing through the diode cannot exceed a certain value called the Child - Langmuir current. This paper is concerned with an extension of this approach, based on the Child-Langmuir asymptotics to magnetized flows [3]. In particular, the value of the space charge limited current is determined when the magnetic field is small (noninsulated diode). Since the arising model could not be solved analytically, it is very important to discover its properties in noninsulated and nearly-insulated cases first.

For better understanding of the discussed mathematical problem and the obtained results with a rising physical effects in vacuum diode, first we need to introduce the description, how it really works.

The excellent description of this processes found in [4] is brought here.

1.1. Description of vacuum diode. The vacuum diode consists of a hot cathode surrounded by a metal anode inside an evacuated enclosure. At suffciently high temperatures electrons are emitted from the cathode and are attracted to the positive anode. Electrons moving from the cathode to the anode constitute a current; they do

${ }^{*}$ This work is supported by INTAS No. 2000-15 and grant of the National University of Colombia. 
so when the anode is positive with respect to the cathode. When the anode is negative with respect to the cathode, electrons are repelled by the anode and the reverse current is almost zero (due to the tail of the Maxwellian distribution of the electrons it is greater than zero). The space between the anode and the cathode is evacuated, so that electrons may move between the electrodes unimpeded by collisions with gas molecules. If $V_{f}=0$ and no emission takes place, the diode may be regarded as a parallel-plate capacitor whose potential difference is $V_{p}$. In this case, the potential distribution in the cathode-plate space is represented by a straight line which joins the points corresponding to cathode potential $V_{k}=0$ and the plate potential $V_{p}$. When the filament voltage rises, the electrons leaving the cathode gang up in the interelectrode space as a cloud called a space charge. This charge alters the potential distribution. Since the electrons making up the space charge are negative, the potential in the cathode-plate space goes up, though all points remain at positive potential. The vector of the electric field is directed from the plate to the cathode, so all the electrons escaping from the cathode make for the plate. In this case, the plate current equals the emission current. One could say the all electrons are being sucked away from the cathode by the anode. This region is known as the emission-limited region. As the filament voltage is increased, emission increases, and so does the space charge. Electrons having low initial velocities are driven back to the cathode by the negative space charge due to the electrons. The density of the electron cloud near the cathode increases to the point where it forms a negative potential region whose minimum, $V_{\text {min }}$, is usually within a few hundredth or tenths of a millimetre of the cathode surface. Thus, there is a high retarding electric field near the cathode $\left(0<x<x_{\min }\right)$; the vector is directed away from the cathode to the plate. To overcome this field, an initial velocity $v_{0}$ of the electrons leaving the cathode should exceed a certain value determined by $V_{\min }, v_{0}>\sqrt{2 \frac{e}{m} V_{\min }}$.

If the electron is below this value, the electron will not be able to overcome the potential barrier. It will slow down to a stop, and the field will push it back to the cathode. Accordingly, the retarding field region (from 0 to $x_{\min }$ ) contains not only electrons traveling away from the cathode, but also those falling back towards the cathode. At a constant filament voltage, a dynamic equilibrium sets in, so that the number of electrons reaching the plate and the number falling back to the cathode is equal to the number of electrons emitted by the cathode. Therefore, plate current is smaller than emission current, or the cathode produces more electrons than the anode can.

1.2. Description of the mathematical model. We consider a plane diode consisting of two perfectly conducting electrodes, a cathode $(X=0)$ and an anode $(X=L)$ supposed to be infinite planes, parallel to $(Y ; Z)$. The electrons, with charge $-e$ and mass $m$, are emitted at the cathode and submitted to an applied electromagnetic field $\mathbf{E}_{\text {ext }}=E_{\text {ext }} X$; $\mathbf{B}_{\text {ext }}=B_{\text {ext }} Z$ such that $E_{\text {ext }} \leq 0$ and $B_{\text {ext }} \geq 0$. Such an electromagnetic field does not act on the $P_{Z}$ component of the particle momentum. Hence, we shall consider a situation where this component vanishes, leading to a confinement of electrons to the plane $Z=0$. The relationship between momentum and velocity is then given by the relativistic relations 


$$
\begin{gathered}
\mathbf{V}(\mathbf{P})=\frac{\mathbf{P}}{\gamma m}, \quad \gamma=\sqrt{1+\frac{|\mathbf{P}|^{2}}{m^{2} c^{2}}}, \\
\mathbf{V}=\left(V_{X}, V_{Y}\right), \quad \mathbf{P}=\left(P_{X}, P_{Y}\right), \quad|\mathbf{P}|^{2}=P_{X}^{2}+P_{Y}^{2},
\end{gathered}
$$

which can also be written

$$
\mathbf{V}(\mathbf{P})=\nabla_{P} \mathcal{E}(\mathbf{P}),
$$

where $\mathcal{E}$ is the relativistic kinetic energy

$$
\mathcal{E}(\mathbf{P})=m c^{2}(\gamma-1),
$$

and $c$ is the speed of light. We shall moreover assume that the electron distribution function $F$ does not depend on $Y$ and that the flow is stationary and collisionless. The injection profile $G\left(P_{X}, P_{Y}\right)$ at the cathode is assumed to be given whereas no electron is injected at the anode. The system is then described by the so called 1,5 dimensional Vlasov-Maxwell model

$$
\begin{gathered}
V_{X} \frac{\partial F}{\partial X}+e\left(\frac{d \Phi}{d X}-V_{Y} \frac{\partial F}{\partial P_{X}}\right)+e V_{X} \frac{d A}{d X} \frac{\partial F}{\partial P_{Y}}=0, \\
\frac{d^{2} \Phi}{d X^{2}}=\frac{e}{\varepsilon_{0}} N(X), \quad X \in(0, L), \\
\frac{d^{2} A}{d X^{2}}=-\mu_{0} J_{Y}(X), \quad X \in(0, L),
\end{gathered}
$$

subject to the following boundary conditions :

$$
\begin{gathered}
F\left(0, P_{X}, P_{Y}\right)=G\left(P_{X}, P_{Y}\right), \quad P_{X}>0, \\
F\left(L, P_{X}, P_{Y}\right)=0, \quad P_{X}<0, \\
\Phi(0)=0, \quad \Phi(L)=\Phi_{L}=-L E_{\mathrm{ext}}, \\
A(0)=0, \quad A(L)=A_{L}=L B_{\text {ext }} .
\end{gathered}
$$

In this system, the macroscopic quantities, namely the particle density $N, X$ and $Y$ are the components of the current density $J_{X}, J_{Y}$. In the above equations, $\varepsilon_{0}$ and $\mu_{0}$ are respectively the vacuum permittivity and permeability.

The boundary conditions are justified by the fact that the electric field $E=-d \frac{\Phi}{d X}$ and the magnetic field $B=-\frac{d A}{d X}$ are exactly equal to the external fields when selfconsistent effects are ignored $\left(N=J_{Y}=0\right)$.

The 1,5 dimensional model (4)-(10) ignores the self-consistent magnetic field due to $J_{X}$, which would introduce two-dimensional effects, and is only an approximation of the complete stationary Vlasov-Maxwell system. In this paper we especially interested in the case, when the applied magnetic field is not strong enough to insulate the diode, $J_{X}$ does not vanish and our model can be viewed as an approximation of the Maxwell equations. 
In order to get a better insight in the behaviour of the diode, we write the model in dimensionless variables in the spirit of $[2,5]$. We first introduce the following units respectively for position, velocity, momentum, electrostatic potential, vector potential, particle density, current and distribution function:

$$
\begin{gathered}
\bar{X}=L, \quad \bar{V}=c, \quad \bar{P}=m c, \quad \mathcal{E}=m c^{2}, \\
\bar{\Phi}=\frac{m c^{2}}{e}, \quad \bar{A}=\frac{m c}{e}, \quad \bar{N}=\frac{\varepsilon_{0} \bar{\Phi}}{x \bar{X}^{2}}, \quad \bar{J}=-e c \bar{N}, \quad \bar{F}=\frac{\bar{N}}{\bar{P}^{2}},
\end{gathered}
$$

and the corresponding dimensionless variables

$$
\begin{gathered}
x=\frac{X}{\bar{X}}, \quad \mathbf{p}=\frac{\mathbf{P}}{\bar{P}}=\left(p_{x}, p_{y}\right), \\
\mathbf{v}=\left(v_{x}, v_{y}\right)=\frac{\mathbf{V}}{\bar{V}}=\frac{\mathbf{p}}{\sqrt{1+\mathbf{p}^{2}}}, \quad \epsilon=\frac{\mathcal{E}}{\overline{\mathcal{E}}}=\sqrt{1+\mathbf{p}^{2}}-1, \\
\varphi=\frac{\Phi}{\bar{\Phi}}, \quad a=\frac{A}{\bar{A}}, \quad n=\frac{N}{\bar{N}}, \quad \mathbf{j}=\frac{\mathbf{J}}{\bar{J}}, \quad f=\frac{F}{\bar{F}} .
\end{gathered}
$$

The next step is to express that particle emission at the cathode occurs in the ChildLangmuir regime: in such a situation, the thermal velocity $V_{G}$ is much smaller than the typical drift velocity supposed to be of the order of the speed of light $c$. Letting $\varepsilon=V_{G} / c$, we shall assume that

$$
f\left(0, p_{x}, p_{y}\right)=g^{\varepsilon}\left(p_{x}, p_{y}\right)=\frac{1}{\varepsilon^{3}} g\left(\frac{p_{x}}{\varepsilon}, \frac{p_{y}}{\varepsilon}\right), \quad p_{x}>0,
$$

where $g$ is a given profile. The scaling factor $\varepsilon^{3}$ insures that the incoming current remains finite independently of $\varepsilon$, whereas the dependence on $\frac{p}{\varepsilon}$ expresses the fact that electrons are emitted at the cathode with a very small velocity. We refer to $[2,5]$ for a detailed discussion of the scaling. The dimensionless system reads

$$
\begin{gathered}
v_{x} \frac{\partial f^{\varepsilon}}{\partial x}+\left(\frac{d \varphi^{\varepsilon}}{d x}-v_{y} \frac{d a^{\varepsilon}}{d x}\right) \frac{\partial f^{\varepsilon}}{\partial p_{x}}+v_{x} \frac{d a^{\varepsilon}}{d x} \frac{\partial f^{\varepsilon}}{\partial p_{y}}=0, \\
\left(x, p_{x}, p_{y}\right) \in(0,1) \times \mathbb{R}^{2}, \\
\frac{d^{2} \varphi^{\varepsilon}}{d x^{2}}=n^{\varepsilon}(x), \quad x \in(0,1), \\
\frac{d^{2} a^{\varepsilon}}{d x^{2}}=j_{y}^{\varepsilon}(x), \quad x \in(0,1) .
\end{gathered}
$$

Here $n^{\varepsilon}(x)$ is a particle density, $j_{y}^{\varepsilon}(x)$ is a current density in $Y$ direction. The initial and boundary conditions are also transformed

$$
\begin{gathered}
f^{\varepsilon}\left(0, p_{x}, p_{y}\right)=g^{\varepsilon}\left(p_{x}, p_{y}\right)=\frac{1}{\varepsilon^{3}} g\left(\frac{p_{x}}{\varepsilon}, \frac{p_{y}}{\varepsilon}\right), \quad p_{x}>0, \\
f^{\varepsilon}\left(1, p_{x}, p_{y}\right)=0, \quad p_{x}<0, \\
\varphi^{\varepsilon}(0)=0, \quad \varphi^{\varepsilon}(1)=\varphi_{L},
\end{gathered}
$$




$$
a^{\varepsilon}(0)=0, \quad a^{\varepsilon}(1)=\varphi_{L} .
$$

Omitting the complete derivation of the limit system, when $\varepsilon \rightarrow 0$, we need to introduce some notions and notations used ahead.

Definition 1. We define $\theta(x)=(1+\varphi(x))^{2}-1-a^{2}(x)$ as an effective potential.

It is readily seen that electrons do not enter the diode unless the effective potential $\theta$ is nonnegative in the vicinity of the cathode. Therefore, we always have $\theta^{\prime}(0) \geq 0$. The limiting case $\theta^{\prime}(0)=0$ is the space charge limited or the Child-Langmuir regime. In view (16), (17) (it still hold in the limit $\varepsilon \rightarrow 0$ ), this condition is equivalent to the standard Child-Langmuir condition $\frac{d \varphi}{d x}(0)=0$.

Let $\theta_{L}$ be the value of $\theta$ at the anode $\theta_{L}=\left(1+\varphi_{L}\right)^{2}-1-a_{L}^{2}$. If $\theta_{L}<0$, electrons cannot reach the anode $x=1$, they are reflected by the magnetic forces back to the cathode and the diode is said to be magnetically insulated. This enables us to define the Hull cut-off magnetic field, which is the relativistic version of the critical field introduced in [6] in the nonrelativistic case:

$$
a_{L}^{H}=\sqrt{\varphi_{L}^{2}+2 \varphi_{L}}
$$

The diode is magnetically insulated if $a_{L}>a_{L}^{H}$, and is not insulated if $a_{L}<a_{L}^{H}$ In dimensional variables, the Hull cut-off magnetic field is given by

$$
B^{H}=\frac{1}{L c} \sqrt{\Phi_{L}^{2}+\frac{2 m c^{2}}{e} \Phi_{L}} .
$$

Thus our primary goal is a stugy of noninsulated, or nearly insulated diodes, which means $B_{\text {ext }}<B^{H}$. The complete derivation of the model is given in [3], while we need only its formal expressions

$$
\begin{aligned}
& \frac{d^{2} \varphi}{d x^{2}}(x)=j_{x} \frac{1+\varphi(x)}{\sqrt{(1+\varphi(x))^{2}-1-a^{2}(x)}}, \\
& \frac{d^{2} a}{d x^{2}}(x)=j_{x} \frac{a(x)}{\sqrt{(1+\varphi(x))^{2}-1-a^{2}(x)}},
\end{aligned}
$$

with a corresponding Cauchy and boundary conditions

$$
\begin{gathered}
\varphi(0)=0, \quad \varphi(1)=\varphi_{L}, \\
\frac{d \varphi}{d x}(0)=0, \\
a(0)=0, \quad a(1)=a_{L} .
\end{gathered}
$$

Let us recall that the unknowns are the electrostatic potential $\varphi$, the magnetic potential $a$ and the current $j_{x}$ (which does not depend on $x$ ).

It is to be noticed that the whole construction of this model depends heavily on the assumption that the effective potential is positive. Actually, $\theta$ could vanish at some points in the diode, leading to closed trajectories and trapped particles. 


\section{Solution trajectory, upper and lower solutions.}

Finally, the limit model of magnetically noninsulation diode is described by the system of two second order ordinary differential equations (18), (19) with conditions (20) $-(22)$.

2.1. Existence of semitrivial solutions for the problem. Let us introduce the definition of cone in a Banach space $X$.

Definition 2. Let $X$ be a Banach space. A nonempty convex closed set $P \subset X$ is called a cone, if it satisfies the conditions:

(i) $x \in P, \lambda \geq 0$ implies $\lambda x \in P$;

(ii) $x \in P,-x \in P$ implies $x=\mathcal{O}$, where $\mathcal{O}$ denotes zero element of $X$.

Here $\leq$ is the order in $X$ induced by $P$, i.e., $x \leq y$ if and only if $y-x$ is an element of $P$.

We will also assume that the cone $P$ is normal in $X$, i.e., order intervals are norm bounded.

In $X \equiv\left\{(u, v): u, v \in C^{1}(\bar{\Omega}), u=v=0\right\}$ we introduce the norm $|U|_{X}=$ $=|u|_{C^{1}}+|v|_{C^{1}}$, and the norm $|U|_{X}=|u|_{\infty}+|v|_{\infty}$ in $C$, where $U=(u, v)$. Here a cone $P$ is given by $P=\{(u, v) \in X: u \geq 0, v \geq 0$ for all $x \in \Omega\}$. So, if $u \neq 0$, $v \neq 0$ belong to $P$, then $-u,-v$ does not belong. We will work with classical spaces on the intervals $\bar{I}=[a, b], \hat{I}=] a, b], I=(a, b)$ :

$C(\bar{I})$ with norm $\|u\|_{\infty}=\max \{|u(x)|: x \in \bar{I}\}$;

$C^{1}(\bar{I})=\|u\|_{\infty}+\left\|u^{\prime}\right\|_{\infty}$;

$C_{\mathrm{loc}}(I)$, which contains all functions that are locally absolutely continuous in $I$. We introduce a space $C_{\text {loc }}(I)$ because the limit problem is singular for $\varphi=0$. The order $\leq$ in cone $P$ is understood in the weak sense, i.e., $y$ is increasing if $a \leq b$ implies $y(a) \leq y(b)$ and $y$ is decreasing if $a \leq b$ implies $y(a) \geq y(b)$.

Theorem 1 (comparison principle in cone). Let $y \in C(\bar{I}) \cap C_{\mathrm{loc}}(I)$. The function $f$ is defined on $I \times R$. Let $f(x, y)$ is increasing in y function, then

$$
v^{\prime \prime}-f(x, v) \geq w^{\prime \prime}-f(x, w) \text { in mean on } I,
$$

$v(a) \leq w(a), v(b) \leq w(b)$ implies $v \leq w$ on $\bar{I}$.

For the convenience of defining an ordering relation in cone $P$, we make a transformation for the problem (18)-(22). Let $F(\varphi, a)$ and $G(\varphi, a)$ be defined by (18)-(22). Then through the transformation $\varphi=-u$ the limit problem is reduced to the form

$$
\begin{aligned}
-\frac{d^{2} u}{d x^{2}} & =j_{x} \frac{1-u}{\sqrt{(1-u)^{2}-1-a^{2}}} \triangleq \tilde{F}\left(j_{x}, u, a\right), & u(0)=0, & u(1)=\varphi_{L}, \\
\frac{d^{2} a}{d x^{2}} & =j_{x} \frac{a}{\sqrt{(1-u)^{2}-1-a^{2}}} \triangleq \tilde{G}\left(j_{x}, u, a\right), & a(0)=0, & a(1)=a_{L} .
\end{aligned}
$$

We note that all solutions of the initial problem, as well the problem (24), are symmetric with respect to the transformation of sign for the magnetic potential $a:(\varphi, a)=(\varphi,-a)$ or the same $(u, a)=(u,-a)$. Thus we must search only positive solutions $\varphi>0$, $a>0$ in cone $P$ or only negative ones: $\varphi<0, a<0$. Thanks to the symmetry of problem it is equivalently and does not yields the extension of the types of sign-defined solutions of the problem (18)-(22) (respect. (24)). Once more, we note that introduction of negative electrostatic potential in problem (24) is connected with more convenient 
relation between order in cone and positiveness of Green function for operator $-u^{\prime \prime}$ that we use below.

Definition 3. A pair $\left[\left(\varphi_{0}, a_{0}\right),\left(\varphi^{0}, a^{0}\right)\right]$ is called

a) sub-super solution of the problem (18)-(22) relative to $P$, if the following conditions are satisfied:

$$
\begin{gathered}
\left(\varphi_{0}, a_{0}\right) \in C_{\mathrm{loc}}(I) \cap C(\bar{I}) \times C_{\mathrm{loc}}(I) \cap C(\bar{I}), \\
\left(\varphi^{0}, a^{0}\right) \in C_{\mathrm{loc}}(I) \cap C(\bar{I}) \times C_{\mathrm{loc}}(I) \cap C(\bar{I}), \\
\varphi_{0}^{\prime \prime}-j_{x} \frac{1+\varphi_{0}}{\sqrt{\left(1+\varphi_{0}\right)^{2}-1-a^{2}}} \triangleq F\left(\varphi_{0}, a\right) \leq 0 \quad \text { in } \quad I, \\
\left(\varphi^{0}\right)^{\prime \prime}-j_{x} \frac{1+\varphi^{0}}{\sqrt{\left(1+\varphi^{0}\right)^{2}-1-a^{2}}} \triangleq F\left(\varphi^{0}, a\right) \geq 0 \quad \text { in } \quad I \quad \forall a \in\left[a_{0}, a^{0}\right] ; \\
a_{0}^{\prime \prime}-j_{x} \frac{a_{0}}{\sqrt{(1+\varphi)^{2}-1-a_{0}^{2}}} \triangleq G\left(\varphi, a_{0}\right) \leq 0 \quad \text { in } \quad I, \\
\left(a^{0}\right)^{\prime \prime}-j_{x} \frac{a^{0}}{\sqrt{(1+\varphi)^{2}-1-\left(a^{0}\right)^{2}}} \triangleq G\left(\varphi, a^{0}\right) \geq 0 \quad \text { in } \quad I \quad \forall \varphi \in\left[\varphi_{0}, \varphi^{0}\right] ; \\
\varphi_{0} \leq \varphi^{0}, \quad a_{0} \leq a^{0} \quad \text { in } \quad I \quad
\end{gathered}
$$

and on the boundary

$$
\begin{array}{ll}
\varphi_{0}(0) \leq 0 \leq \varphi^{0}(0), & \varphi_{0}(1) \leq \varphi_{L} \leq \varphi^{0}(1), \\
a_{0}(0) \leq 0 \leq a^{0}(0), & a_{0}(1) \leq a_{L} \leq a^{0}(1) ;
\end{array}
$$

b) sub-sub solution of the problem (18)-(22) relative to $P$, if a condition (25) is satisfied and

$$
\begin{aligned}
& \varphi_{0}^{\prime \prime}-F\left(j_{x}, \varphi_{0}, a_{0}\right) \leq 0 \text { in } I, \\
& a_{0}^{\prime \prime}-G\left(j_{x}, \varphi_{0}, a_{0}\right) \leq 0 \text { in } I
\end{aligned}
$$

and on the boundary

$$
\varphi_{0}(0) \leq 0, \quad \varphi_{0}(1) \leq \varphi_{L}, \quad a_{0}(0) \leq 0, \quad a_{0}(1) \leq a_{L} .
$$

Remark 1. In Definition 3 the expressions with square roots we take by modulus of effective potential $\theta(\cdot)$.

By analogy with (26), (27), we may introduce the definition of super-super solution in cone.

Definition 4. The functions $\Phi\left(x, x_{a_{i}}, j_{x}\right), \Phi_{1}\left(x, x_{\varphi_{j}}, j_{x}\right)$ we shall call a semitrivial solutions of the problem (18)-(22), if $\Phi\left(x, x_{a_{i}}, j_{x}\right)$ is a solution of the scalar boundaryvalue problem

$$
\begin{aligned}
\varphi^{\prime \prime}=F\left(j_{x}, \varphi, x_{a_{i}}\right) & =j_{x} \frac{1+\varphi}{\sqrt{(1+\varphi)^{2}-1-\left(x_{a_{i}}\right)^{2}}} \\
\varphi(0) & =0, \quad \varphi(1)=\varphi_{L}
\end{aligned}
$$


and $\Phi_{1}\left(x, x_{\varphi_{j}}, j_{x}\right)$ is a solution of the scalar boundary-value problem

$$
\begin{gathered}
a^{\prime \prime}=G\left(j_{x}, x_{\varphi_{j}}, a\right)=j_{x} \frac{a}{\sqrt{\left(1+x_{\varphi_{j}}\right)^{2}-1-a^{2}}}, \\
a(0)=0, \quad a(1)=a_{L} .
\end{gathered}
$$

Here $x_{a_{i}}, i=1,2,3$, and $x_{\varphi_{j}}, j=1,2$, are respectively, the indicators of semitrivial solutions $\Phi\left(x, x_{a_{i}}, j_{x}\right), \Phi_{1}\left(x, x_{\varphi_{j}}, j_{x}\right)$ defined by the following way:

$$
x_{a_{1}}=0, \quad \text { if } a(x)=0 ;
$$

$x_{a_{2}}=a^{0}, \quad$ if $\quad a=a^{0}$ quadbe upper solution of the problem (29);

$x_{a_{3}}=a_{0}, \quad$ if $\quad a=a_{0}$ quadbe lower solution of the problem (29);

$x_{\varphi_{1}}=\varphi^{0}, \quad$ if $\varphi=\varphi^{0}$ quadbe upper solution of the problem (28);

$x_{\varphi_{2}}=\varphi_{0}, \quad$ if $\varphi=\varphi_{0}$ quadbe upper solution of the problem (28).

From Definition 4, we obtain the following types of scalar boundary-value problems for semitrivial (in sense of Definition 4) solutions are

$$
\begin{aligned}
& \left(A_{1}\right) \quad \varphi^{\prime \prime}=F(\varphi, 0)=j_{x} \frac{1+\varphi}{\sqrt{(1+\varphi)^{2}-1}}, \quad \varphi(0)=0, \quad \varphi(1)=\varphi_{L} \\
& \left(A_{2}\right) \varphi^{\prime \prime}=F\left(\varphi, a^{0}\right)=j_{x} \frac{1+\varphi}{\sqrt{(1+\varphi)^{2}-1-\left(a^{0}\right)^{2}}}, \quad \varphi(0)=0, \quad \varphi(1)=\varphi_{L} \\
& \left(A_{3}\right) \varphi^{\prime \prime}=F\left(\varphi, a_{0}\right)=j_{x} \frac{1+\varphi}{\sqrt{(1+\varphi)^{2}-1-\left(a_{0}\right)^{2}}}, \quad \varphi(0)=0, \quad \varphi(1)=\varphi_{L}, \\
& \left(A_{4}\right) a^{\prime \prime}=G\left(\varphi^{0}, a\right)=j_{x} \frac{a}{\sqrt{\left(1+\varphi^{0}\right)^{2}-1-a^{2}}}, a(0)=0, \quad a(1)=a_{L}, \\
& \left(A_{5}\right) a^{\prime \prime}=G\left(\varphi_{0}, a\right)=j_{x} \frac{a}{\sqrt{\left(1+\varphi_{0}\right)^{2}-1-a^{2}}}, a(0)=0, \quad a(1)=a_{L} .
\end{aligned}
$$

We shall find the solutions of problems $\left(A_{1}\right)-\left(A_{3}\right)$ for $\varphi_{0}<\varphi^{0}$, where $\varphi_{0}\left(x_{a_{1}}\right)$, $\varphi^{0}\left(x_{a_{2}}\right)$ are respectively, lower and upper solutions of problem $\left(A_{1}\right)$. The solution $(\varphi, a)$ of the initial problem should be belong to the interval

$$
\begin{gathered}
\varphi \in \Phi(\varphi, 0) \bigcap \Phi\left(\varphi, a^{0}\right) \bigcap \Phi\left(\varphi, a_{0}\right), \\
a \in \Phi_{1}\left(\varphi^{0}, a\right) \bigcap \Phi_{1}\left(\varphi_{0}, a\right) .
\end{gathered}
$$

Moreover, the ordering of lower and upper solutions of problems $\left(A_{1}\right)-\left(A_{3}\right)$ is satisfied

$$
\varphi_{0}\left(x_{a_{1}}\right)<\varphi_{0}\left(x_{a_{2}}\right)<\varphi_{0}\left(x_{a_{3}}\right)<\varphi^{0}\left(x_{a_{2}}\right)<\varphi^{0}\left(x_{a_{1}}\right) .
$$

We shall seek the solution of problems $\left(A_{4}\right),\left(A_{5}\right)$ for $a_{0}<a^{0}$. In this case the following ordering of lower and upper solutions of problems $\left(A_{4}\right),\left(A_{5}\right)$ :

$$
a_{0}\left(x_{\varphi_{1}}\right)<a_{0}\left(x_{\varphi_{2}}\right)<a^{0}\left(x_{\varphi_{2}}\right)<a^{0}\left(x_{\varphi_{1}}\right)
$$

is satisfied.

We go over to the direct study of the problem (28) which includes the cases $\left(A_{1}\right)-$ $\left(A_{3}\right)$. Let us consider the boundary-value problem (28) with 
$\left(B_{1}\right) F(x, \varphi):(0,1] \times(0, \infty) \rightarrow(0, \infty)$.

In condition $\left(B_{1}\right)$ for $F(x, \varphi)$ we dropped index $a_{i}$, considering a general case of nonlinear dependence $F$ of $x$.

We shall assume that $F$ is a Caratheodory function, i.e.,

$\left(B_{2}\right) F(\cdot, s)$ measurable for all $s \in R$,

$\left(B_{3}\right) F(x, \cdot)$ is continuous a.e. for $\left.\left.x \in\right] 0,1\right]$,

and the following conditions hold:

$\left(B_{4}\right) \int_{0}^{1} s(1-s) F d s<\infty$,

$\left(B_{5}\right) \frac{\partial F}{\partial \varphi}>0, \quad$ i.e., $\quad F \quad$ is increasing in $\varphi$.

There are $\left.\left.\gamma(x) \in L^{1}(] 0,1\right]\right)$ and $\alpha \in R, 0<\alpha<1$ such that

$\left.\left.\left(B_{6}\right) \quad|F(x, s)| \leq \gamma(x)\left(1+|s|^{-\alpha}\right) \quad \forall(x, s) \in\right] 0,1\right] \times R$.

We are intersted in a positive classical solution of equation (28), i.e., $\varphi>0$ in $P$ for $x \in] 0,1]$ and $\left.\left.\varphi \in C([0,1]) \bigcap C^{2}(] 0,1\right]\right)$. The problem (28) is singular, therefore, condition $\left(B_{1}\right)$ is not fulfilled on the interval $\varphi \in(0, \infty)$ and in this connection, the well-known theorems on existence of lower and upper solution in cone $P$ does not work. It follows from Theorem 1 , since $F$ in (28) is increasing in $\varphi$, then $\varphi<w$ for $x \in] 0,1$ ], where $\varphi$ and $w$ satisfy the differential inequality (23).

Theorem 2. Assume conditions $\left(B_{2}\right)-\left(B_{6}\right)$. Then there exists a positive solution $\left.\left.\varphi \in C([0,1]) \bigcap C^{2}(] 0,1\right]\right)$ of the boundary-value problem (28).

Application of monotone iteration techniques to the equation (28) gives an existence of maximal solution $\bar{\varphi}\left(x, j_{x}\right)$ such that

$$
\left.\left.\varphi\left(x, x_{j}\right) \leq \bar{\varphi}\left(x, x_{j}\right)<w(x) \quad \text { for } \quad x \in\right] 0,1\right] .
$$

Proposition 1. Let $0<c \leq j_{x} \leq j_{x}^{\max }$. Then equation $\left(A_{1}\right)$

$$
\begin{gathered}
\varphi^{\prime \prime}=F\left(j_{x}, \varphi, 0\right)=j_{x} \frac{1+\varphi}{\sqrt{\varphi(2+\varphi)}}, \\
\varphi(0)=0, \quad \varphi(1)=\varphi_{L}
\end{gathered}
$$

has a lower positive solution

$$
u_{0}=\delta^{2} x^{4 / 3},
$$

if

$$
4 \delta^{3} \geq 9 j_{x}^{\max }\left(1+\delta^{2}\right) / \sqrt{2+\delta^{2}}
$$

and an upper positive solution

$$
u^{0}=\alpha+\beta x, \quad \alpha, \beta>0,
$$

with 


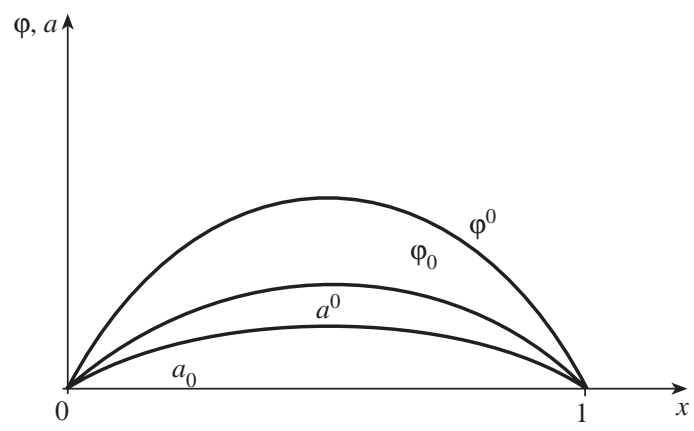

Fig. 1. Location of lower $\left(\varphi_{0}, a_{0}\right)$ and upper $\left(\varphi^{0}, a^{0}\right)$ solutions.

$$
\varphi_{L} \geq \delta^{2}
$$

where $\delta$ is defined from (31).

Remark 2. Square root is taking as $\sqrt{|\varphi(2+\varphi)|}$ in the case of negative solutions. Here $u^{0}=-\epsilon x$ is an upper solution, and $u_{0}=-2+\epsilon$ is a lower solution $(0<\epsilon<$ $<1)$. Hence equation $\left(A_{1}\right)$ has the negative solution only for $0<\varphi_{L}<-2$ because $F(x,-2)=-\infty$.

It follows from (31), (33) that a value of current is limited by the value of electrostatic potential on the anode $\varphi_{L}$

$$
j_{x} \leq j_{x}^{\max } \leq \mathcal{F}\left(\varphi_{L}\right) .
$$

Analysis of lower and upper solutions (30), (32) exhibits that for $\delta^{2}=\varphi_{L}>2$ and $\alpha=\beta \leq 1$ interval in $x$ between lower and upper solutions is decreased, and for the large values of the potential $\varphi_{L}$ diode makes on regime $\varphi_{L} x^{4 / 3}$.

Proposition 2. Let $0<c \leq j_{x} \leq j_{x}^{\max }$. Then equation $\left(A_{4}\right)$

$$
a^{\prime \prime}=G\left(j_{x}, \varphi^{0}, a\right)=j_{x} \frac{a}{\sqrt{\left(1+\varphi^{0}\right)^{2}-1-a^{2}}}, \quad a(0)=0, \quad a(1)=a_{L},
$$

with a lower solution $a_{0}=0$ and an upper solution $a^{0}=u^{0}>0$, has an unique solution $a\left(x, j_{x}, c\right)$, which is positive, moreover

$$
0 \leq a_{L} \leq \sqrt{\varphi^{0}(2+\varphi)}
$$

Remark 3. The problem $\left(A_{5}\right)$ is considered by analogy with problem $\left(A_{4}\right)$, change of an upper solution $a^{0}=u^{0}$ to a lower $a^{0}=u_{0}$ one and $0 \leq a_{L} \leq \sqrt{\varphi_{0 L}\left(2+\varphi_{0 L}\right)}$.

Following to the Definition 3 and Propositions 1, 2, solutions of the problems (28), (29) we can write in the form (Fig. 1)

lower-lower $\left.\left(\varphi_{0}, a_{0}\right)\right)$ :

$$
\varphi_{0}=u_{0}=\delta^{2} x^{4 / 3}, \quad a_{0}=0, \quad \varphi_{L} \geq \delta^{2} ;
$$

upper-lower $\left(\varphi^{0}, a_{0}\right)$ :

$$
\varphi^{0}=u^{0}=\alpha+\beta x, \quad a_{0}=0, \quad \delta^{2} \leq \varphi_{L} \leq \mathcal{C}, \quad \mathcal{C}=\max \{\alpha, \beta\} ;
$$

lower-upper $\left(\varphi_{0}, a^{0}\right)$ :

$$
\varphi_{0}=u_{0}=\delta^{2} x^{4 / 3}, \quad a^{0}=u^{0}, \quad \varphi_{L} \geq \delta^{2}, \quad a_{L} \leq \sqrt{\left(u_{0}\left(2+u_{0}\right)\right.} ;
$$



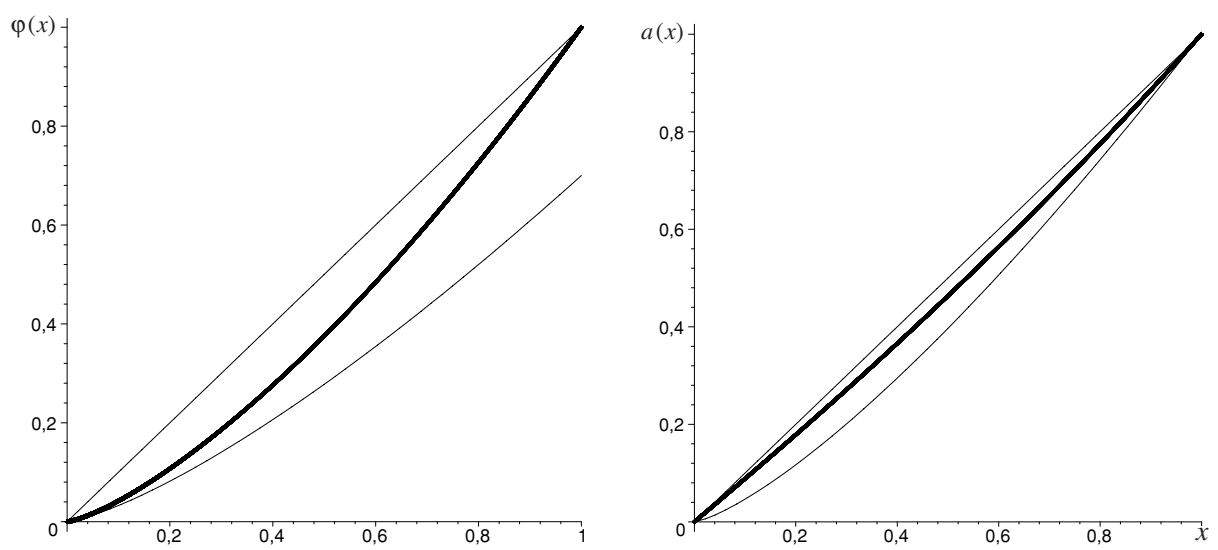

Fig. 2. Numerical solution for $\varphi_{L}=a_{L}=1$; estimated $j_{x}=0,534075023488271$, $\frac{d a}{d x}(0)=0,879738089874635$. Function $\varphi(x)$ : upper solution $y=x$ and lower solution $y=\frac{7}{10} x^{\frac{4}{3}}$. Function $a(x)$ : upper solution $y=x$ and lower solution $y=x^{\frac{4}{3}}$.
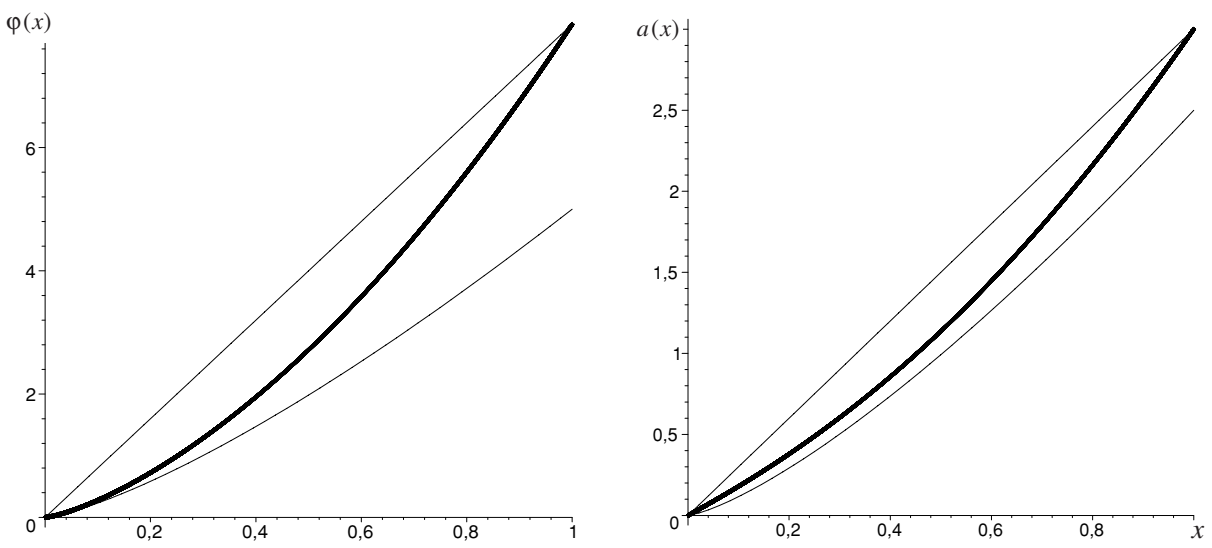

Fig. 3. Numerical solution for $\varphi_{L}=8, a_{L}=3$; estimated $j_{x}=8,93859989164142$, $\frac{d a}{d x}(0)=1,72776197665836$. Function $\varphi(x)$ : upper solution $y=8 x$ and lower solution $y=5 x^{\frac{4}{3}}$. Function $a(x)$ : upper solution $y=3 x$ and lower solution $y=\frac{5}{2} x^{\frac{4}{3}}$.

upper-upper $\left(\varphi^{0}, a^{0}\right)$ :

$$
\varphi^{0}=u^{0}=\alpha+\beta x, \quad a^{0}=u^{0}, \quad \varphi_{L} \leq \mathcal{C}, a_{L} \leq a^{0} \leq u^{0} .
$$

Thus we have the following main result:

Theorem 3. Assume conditions $\left(B_{2}\right),\left(B_{3}\right),\left(B_{6}\right)$ and inequalities (31), (32) and

$$
a_{L} \leq \frac{j_{x}}{2} \leq \frac{j_{x}^{\max }}{2} \leq \frac{\mathcal{F}\left(\varphi_{L}\right)}{2}
$$

fulfilled. Then the problem (18)-(22) possesses a positive solution in cone P such that

$$
\begin{gathered}
\varphi_{0}^{\prime \prime} \geq j_{x} F\left(\varphi_{0}, z_{2}\right), \quad z_{2} \in\left[0, \varphi^{0}\right], \\
\left(\varphi^{0}\right)^{\prime \prime} \leq j_{x} F\left(\varphi^{0}, z_{2}\right), \quad z_{2} \in\left[0, \varphi^{0}\right], \\
a_{0}^{\prime \prime} \geq G\left(j_{x}, z_{1}, a_{0}\right), \quad z_{1} \in\left[\varphi_{0}, \varphi^{0}\right], \\
\left(a^{0}\right)^{\prime \prime} \leq G\left(j_{x}, z_{1}, a^{0}\right), \quad z_{1} \in\left[\varphi_{0}, \varphi^{0}\right],
\end{gathered}
$$



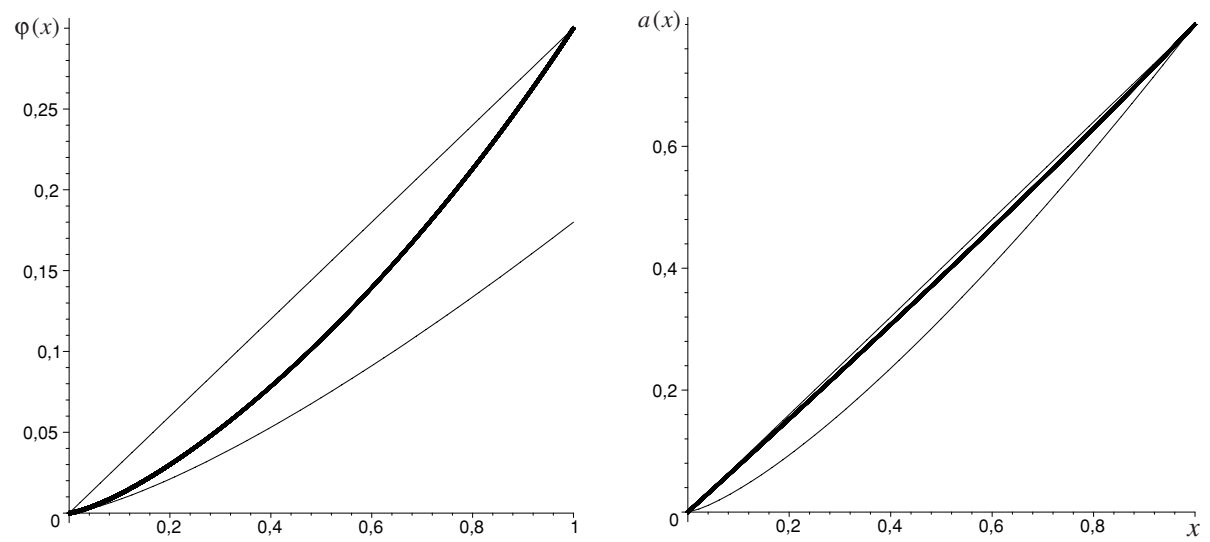

Fig. 4. Numerical solution for $\varphi_{L}=0,3, a_{L}=0,8$; estimated $j_{x}=0,0761231763035411$, $\frac{d a}{d x}(0)=0,759092882499624$. Function $\varphi(x):$ upper solution $y=0,3 x$ and lower solution $y=0,18 x^{\frac{4}{3}}$. Function $a(x)$ : upper solution $y=0,8 x$ and lower solution $y=0,8 x^{\frac{4}{3}}$.

where $\varphi_{0}=\delta^{2} x^{4 / 3}$ is a lower solution of problem $\left(A_{1}\right), \varphi^{0}=\alpha+\beta x, \alpha, \beta>0$, is an upper solution of problem $\left(A_{1}\right)$ with condition $\varphi_{L} \geq \delta^{2} ; a_{0}=0$ is a lower solution of problem $\left(A_{4}\right)$ with condition $0 \leq a_{L} \leq \sqrt{\varphi^{0}\left(2+\varphi^{0}\right)}$.

2.2. Numerical trajectories and the upper and lower solutions. Leaving apart the discussion of numerical solution methods for next the sections, here we provide some numerical solution trajectory examples both for $\varphi(x)$ and $a(x)$ evaluated for different boundary conditions, see Fig. $2-4$. They clearly show that that the theoretical results fully coincide with the obtained numerical estimations.

Acknowlegment. We kindly thank professor Pierre Degond for his remarks and support while professor Alexandr Sinitsyn was staying in Universit'e Paul Sabatier, Toulouse, France where this research paper was finished.

1. I. Langmuir, Compton K.T. Electrical discharges in gases: Pt II. Fundamental phenomena in electrical discharges // Rev. Mod. Phys. - 1931. - 3. - P. 191-257.

2. Degond P., Raviart P.-A. An asymptotic analysis for the one-dimensional Vlasov-Poisson system // Asymptotic Anal. - 1991. - 4. - P. 187-214.

3. Ben Abdallah N., Degond P., M'ehats F. Mathematical models of magnetic insulation // Ibid. -1999 . - 20. - P. $97-132$.

4. Kelly $O$. Shot noise in a diode // El. manuscr. - 1999. - http: \\www.maths.tcd.ie $\backslash$ olly $\backslash$ shot.pdf. P. 11.

5. Degond P., Raviart P.-A. On a penalization of the Child-Langmuir emission condition for the one-dimensional Vlasov- Poisson equation // Asymptotic Anal. - 1992. - 6. - P. 1-27.

6. Hull A. W. The effect of a uniform magnetic field on the motion of electrons between coaxial cylinders // Phys. Rev. - 1921. - 18. - P. 31-57.

Received 26.10.2004 